\title{
Clinical and Socio-Demographic Correlates in Depressive Patients with Suicidal Ideas \& Suicidal Attempts
}

\author{
Dr. Syed Ummer. ${ }^{1 *}$, Dr. Sushith Sugathan. $C^{2}$
}

\section{ABSTRACT}

Background: Suicide has a strong association with mental disorder and contributes to the excess mortality of the mentally ill. Suicidal ideation is prevalent and appears to be a precondition for suicide attempts among psychiatric patients with Major Depressive disorder. Though ideas and attempts may overlap there are studies that show the two are separate clinical entities with unique psycho-socio demographic profile. Aim: To study correlates in patients with suicidal ideation and those with suicide attempts in a cohort of major depressive disorder patients. Methodology: Study was conducted at the Inpatient psychiatry unit at a private medical college in Coimbatore, Tamil Nadu. It was a Cross sectional study design. Patients consecutively admitted with major depressive disorder (DSM IV TR) were recruited. Severity of suicidal ideations and attempts were rated on validated scales and the socio demographic and clinical correlates were analyzed. Results: Clinical correlates like severity of depression, severity of suicidal ideation and history of past suicidal attempt were positively correlated with severity of suicidal ideation as well as severity of current suicidal attempts in the cohort of depressive disorder patients. Conclusion: Assessment of current episode severity of both suicidal ideation as well as suicidal attempt is important in identifying high risk patients with major depressive disorder.

Keywords: Clinical, Socio-Demographic, Correlates, Depressive Patients, Suicidal Ideas, Suicidal Attempts

According to World health organization (WHO), over 800,000 people die due to suicide every year. Also for every suicide there are many more people who attempt suicide every year. WHO defines suicidal act as the injury with varying degree of lethal intent and suicide as, such acts with fatal outcome? Suicidal acts with no fatal outcome are defined as suicide attempts, attempted suicide, Para suicide, or act of deliberate self-harm.

\footnotetext{
${ }^{1}$ Associate Professor, Dept. of Psychiatry, PSG Institute of Medical Science \& Research, Coimbatore, India

${ }^{2}$ Senior Resident, Dept. of Psychiatry, PSG Institute of Medical Science \& Research, Coimbatore, India *Responding Author
}

Received: January 22, 2017; Revision Received: February 10, 2017; Accepted: February 15, 2017

(C) 2017 Ummer S, Sugathan S; licensee IJIP. This is an Open Access Research distributed under the terms of the Creative Commons Attribution License (www.creativecommons.org/licenses/by/2.0), which permits unrestricted use, distribution, and reproduction in any Medium, provided the original work is properly cited. 


\section{Clinical and Socio-Demographic Correlates in Depressive Patients with Suicidal Ideas \& Suicidal Attempts}

A prior suicide attempt is the single most important risk factor for suicide in the general population. In 2004, suicide was the $16^{\text {th }}$ leading cause of death worldwide. Also suicide is the second leading cause of death among 15-29 year olds and this is an alarming finding. It is not only in the developed countries that suicide has been studied, but $75 \%$ of global suicides occur in low and the middle income countries.

There are several studies that have been done on incidence of suicide in India. Over the years studies have reported incidence rates that range from 2.36 to 42 per 100,000 populations. Majority of these studies have been done in hospital based setting and few in community based samples. In one of the earliest article on attempted suicide by Venkoba Rao, an incidence rate of 43/100,000 in Madurai was reported. Also in the same study, 1 in 12 cases of the suicide attempts were fatal ${ }_{4}$ ). According to the NCRB report in 2010 the rate of suicide was 11.4 per 100,000 populations; an increase of 5.9\% when taking into accounts the rate from the year 2008 onwards.

Suicide has a strong association with mental disorder and contributes to the excess mortality of the mentally ill. A systematic review and meta-analysis, by Harris and Barraclough et al, which included 249 reports on the mortality of mental disorders, comparing the relative risk of suicide for a given psychiatric disorder with the expected suicide rate in the general population, all psychiatric diagnoses (except mental retardation), had an increased mortality rate underscoring the importance of making a correct psychiatric diagnosis in suicide risk assessment.

Suicidal ideation is prevalent and appears to be a precondition for suicide attempts among psychiatric patients with Major Depressive disorder. The overall level of psychopathology of suicide attempters is higher compared with that in patients with ideation. Another important aspect to look at is whether suicide and attempted suicide along with other suicidal behavior form a single entity. Previous studies on suicide and attempted suicide reveals that these two phenomena tended to be regarded as one, directed at death, which succeeded in some cases and failed in others. Whereas there are recent investigators who have suggested that suicide and attempted suicide are two separate clinical entities with unique psycho-socio demographic profile, though there may be some overlap.

In the Weissman et al study they had conducted nine cross-national epidemiological surveys using similar diagnostic assessment and criteria (DSM 3) and reported lifetime prevalence rates per 100 for suicide ideation that ranged from 2.09 (Beirut) to 18.51 (Christchurch, New Zealand). Lifetime prevalence rates per 100 for suicide attempts ranged from 0.72 (Beirut) to 5.93 (Puerto Rico). 


\section{Clinical and Socio-Demographic Correlates in Depressive Patients with Suicidal Ideas \& Suicidal}

Attempts

Other studies have analyzed suicidal behavior and its correlates in specific psychiatric disorder as well. In a study by Srivastava et al they had found a positive correlation between suicide attempt and factors like the severity of depression, male gender, and age less than 35 years, being married, and being employed.

In another study done at Varanasi, on a sample of 60 out-patient psychiatry patients with diagnosis of Major depressive disorder (DSM IV-TR), they reported that all the patients who express suicidal ideation did not attempt suicide. Suicidal attempts were reported in only $16.6 \%$ of patients with major depressive episode with suicidal ideation. Also young patients with depression, unmarried men, married women and students, having severe suicidal ideation with agitation or paranoid symptoms were more likely to attempt suicide.

\section{Rationale for study}

There are no Indian studies comparing the clinical and socio demographic variables with the severity of suicidal ideation and suicide attempt as distinct entities in major depressive disorder. Most of the studies had considered both these groups as a continuum, whereas we want to study these two groups as distinct factors influencing suicidal behavior in depression. Also though many studies had studied correlation of suicidal behavior with severity of depression, they had not analyzed the socio demographic and clinical correlations with the severity of suicidal ideation or severity of attempt.

\section{Aim}

To compare the socio demographic and clinical variables between the patients with suicidal ideation and those with suicide attempt in a cohort of major depressive disorder patients.

\section{Objectives}

1. To compare the correlation of socio demographic and clinical variables with the severity of suicidal ideation.

2. To compare the correlation of socio demographic and clinical variables with the severity of suicidal attempt.

\section{METHODOLOGY}

This study was a cross sectional consecutive sampling study that was conducted in the psychiatry In-patient ward at a private medical college and hospital in Coimbatore, Tamil Nadu. Study duration was 1 year (from January 2016 to December 2016) and all the consecutive patients, between the ages of 18 and 65 years, admitted in the psychiatry ward with the diagnosis of major depressive disorder were screened for study inclusion (Fig. 1). 


\section{Clinical and Socio-Demographic Correlates in Depressive Patients with Suicidal Ideas \& Suicidal Attempts}

After explaining the study protocol, we got written informed consent from the patient and the family member who had accompanied the patient. Consenting patients were interviewed by the researcher.

Inclusion criteria consisted of patients whose age was between 18 and 65 yrs and should have been admitted in the psychiatry ward with diagnosis of Major depressive disorder by their respective consulting psychiatrist in the department who was blind to the study protocol. The study researcher administers the SCID for DSM IV interview schedule and if the diagnosis is confirmed for Major depressive disorder the patient is included in the study.

Exclusion criteria consists of patients who refused to give consent and patients who with mental retardation or neuro-psychiatric co morbidity like dementia were also excluded as they would not be able to accurately respond to the severity assessment scales being administered. Patients with Bipolar depression were excluded from the study as we wanted to study specifically unipolar depressive disorder group of patients. Patients with co morbid physical illness, substance abuse and other psychiatric co morbidities were also excluded from the study. Total sample size for the study was 53.

Fig 1. Flow chart for study recruitment and methodology

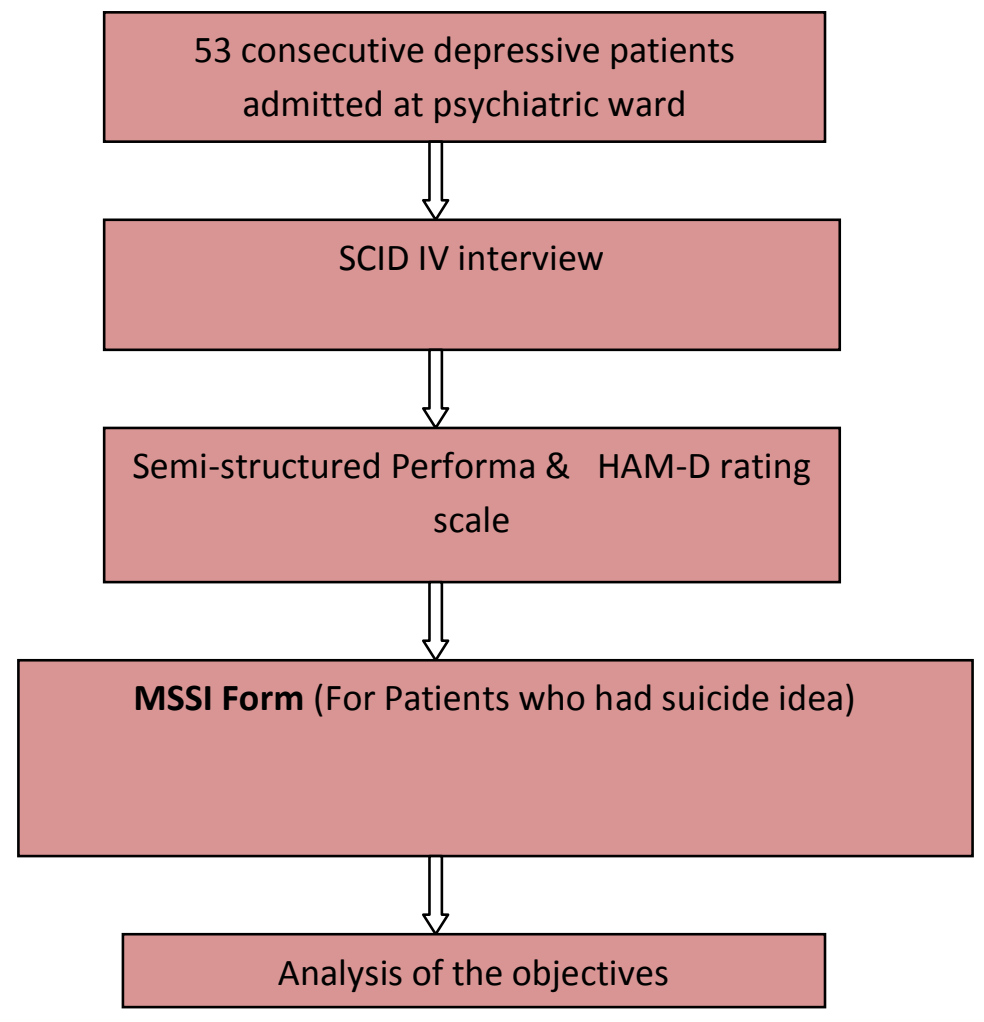




\section{Measurements}

Once the patient was recruited into the study, the researcher had collected details about the socio demographic and clinical variables by using a semi structured profoma. The researcher then administered the Hamilton depression rating scale (HAMD-17) to assess the severity of depression. The researcher then administered the following scales for assessment of severity of suicidal behaviour:

1. Suicide Risk Assessment Form (SRAF)- a scale that was developed in order to assess the severity of suicide attempts. The scale consists of 15 questions which are scaled from $0-2$, which take into account both the logistics of suicide attempt as well as the intent. The scale has high reliability and validity ${ }^{16}$.

2. Modified Scale for Suicidal Ideation (MSSI) - this scale was developed for assessing severity of suicide ideas. Principal component analysis revealed a bi dimensional structure, with factors corresponding to (1) Desire and Ideation and (2) Plans and Preparations. Each factor displayed acceptable internal consistency and expected patterns of convergent validity via associations with hopelessness, depressive symptoms, impulsivity, and a selfreport measure of suicidal behaviors.

\section{Statistical analysis}

We performed statistical analysis with PASW (SPSS) version 19.0 for Windows. The correlation of socio-demographic variables with suicide ideation and depressive patients who attempted suicide, patient history with suicidal attempts, severity of depression with suicidal ideation and suicide attempt were determined using chi-square test. The correlation of suicidal ideation and suicide attempt were determined using Pearson correlation. P-value less than 0.05 were considered as statistically significant.

\section{RESULTS}

\section{Socio-demographic and clinical variables in the whole sample}

Most of the patients belong to early adulthood age group (47.2\%). Out of the total sample of 53 patients, 24 were males and 29 were females. Majority of the patients belong to middle and low socio economic status (90.6\%), were married (75.5\%) residing in rural community (69.8\%) and were graduates (35.8\%) and were living in a nuclear family (71.7\%). Most of the patients did not have family history of suicide (84.9\%).

The mean severity of depression on HAMD-17 rating scale in the sample was $28.72(+/-6.3)$, thus indicating severe depression. $79.2 \%$ of the patients had very severe depressive episode (42/53) as per HAM-D rating scale.

Three-fourth (75\%) of the patients did not have any past suicide attempts. Out of the total patients who had attempted suicide $(\mathrm{N}=13)$, one patient had attempted suicide three times in the 


\section{Clinical and Socio-Demographic Correlates in Depressive Patients with Suicidal Ideas \& Suicidal Attempts}

past (1.9\%) and 12 patients had attempted once (22.6\%). Among 53 recruited depression patients 35 patients (66\%) had suicidal ideas and 13 patients had attempted suicide (24.5\%). Among the patients who had suicidal ideas, majority (62\%) of the patients had severe suicidal ideas as per MSIS scale (i.e, total score $>20$ ). Among patients who did attempt suicide (13/53), majority of them (53.8\%) had high suicide attempt severity (SRAF total score>20), $30.8 \%$ had medium severity and $15.4 \%$ had low severity of suicide attempt.

\section{Correlation of Socio demographic variables with severity of suicidal ideas}

Suicidal ideas were common and were more severe, in age group 18-40 years than middle and late adulthood age group. The severity of suicidal ideas was high among lower socioeconomic status patients and who were married. Unemployment and patients residing in rural background and patients in nuclear families had more severe suicidal ideas. But none the above socio demographic variables were statistically significant when compared across the grades of severity of suicidal ideation (Table 1)

Table 1: Correlation of socio-demographic variables with severity of suicide ideation

\begin{tabular}{|c|c|c|c|c|c|}
\hline & & \multicolumn{3}{|c|}{ SUICIDAL IDEATION SEVERITY } & \multirow[t]{2}{*}{ P-VALUE } \\
\hline & & ABSENT & MILD & SEVERE & \\
\hline \multirow[t]{3}{*}{ AGE (YRS) } & $18-40$ & $6(24.0 \%)$ & $6(24.0 \%)$ & $13(52 \%)$ & 0.256 \\
\hline & $41-60$ & $6(35.3 \%)$ & $6(35.3 \%)$ & $5(29.4 \%)$ & \\
\hline & $61-65$ & $6(54.5 \%)$ & $1(9.1 \%)$ & $4(36.4 \%)$ & \\
\hline \multirow[t]{2}{*}{ GENDER } & MALE & $8(33.8 \%)$ & $4(16.7 \%)$ & $12(50.0 \%)$ & 0.392 \\
\hline & FEMALE & $10(34.5 \%)$ & $9(31.0 \%)$ & $10(34.5 \%)$ & \\
\hline SOCIO & HIGH & $2(40.0 \%)$ & $1(20.0 \%)$ & $2(40.0 \%)$ & 0.699 \\
\hline ECONOMIC & MIDDLE & $9(45.0 \%)$ & $4(20.0 \%)$ & $7(35.0 \%)$ & \\
\hline STATUS & LOW & $7(25.0 \%)$ & $8(28.62 \%)$ & $13(46.4 \%)$ & \\
\hline MARITAL & MARRIED & $14(35.0 \%)$ & $10(25.0 \%)$ & $16(40.0 \%)$ & 0.620 \\
\hline \multirow[t]{2}{*}{ STATUS } & UNMARRIED & $2(20.0 \%)$ & $3(30.0 \%)$ & $5(50.0 \%)$ & \\
\hline & WIDOW & $2(66.7 \%)$ & $0(0.0 \%)$ & $1(33.3 \%)$ & \\
\hline \multirow[t]{3}{*}{ OCCUPATION } & EMPLOYED & $8(36.4 \%)$ & $6(27.3 \%)$ & $8(36.4 \%)$ & 0.834 \\
\hline & $\begin{array}{l}\text { UNEMPLOY } \\
\text { ED }\end{array}$ & $9(34.6 \%)$ & $5(19.2 \%)$ & $12(46.2 \%)$ & \\
\hline & STUDENT & $1(20.0 \%)$ & $2(40.0 \%)$ & $2(40.0 \%)$ & \\
\hline PLACE OF & URBAN & $4(25.0 \%)$ & $3(18.8 \%)$ & $9(56.3 \%)$ & 0.358 \\
\hline LIVING & RURAL & $14(37.8 \%)$ & $10(27.0 \%)$ & $13(35.1 \%)$ & \\
\hline \multirow[t]{4}{*}{ EDUCATION } & ILLITERATE & $4(40.0 \%)$ & $3(30.0 \%)$ & $3(30.0 \%)$ & 0.941 \\
\hline & $\begin{array}{l}\text { MIDDLE } \\
\text { SCHOOL }\end{array}$ & $3(42.9 \%)$ & $1(14.3 \%)$ & $3(42.9 \%)$ & \\
\hline & $\begin{array}{l}\text { HIGH } \\
\text { SCHOOL }\end{array}$ & $3(25.0 \%)$ & $3(25.0 \%)$ & $6(50.0 \%)$ & \\
\hline & $\begin{array}{l}\text { HIGHER } \\
\text { SECONDAR }\end{array}$ & $2(40.0 \%)$ & $2(40.0 \%)$ & $1(20.0 \%)$ & \\
\hline
\end{tabular}

(C) The International Journal of Indian Psychology, ISSN 2348-5396 (e)| ISSN: 2349-3429 (p) | 91 
Clinical and Socio-Demographic Correlates in Depressive Patients with Suicidal Ideas \& Suicidal Attempts

\begin{tabular}{|c|c|c|c|c|c|}
\hline & & \multicolumn{3}{|c|}{ SUICIDAL IDEATION SEVERITY } & \multirow[t]{2}{*}{ P-VALUE } \\
\hline & & ABSENT & MILD & SEVERE & \\
\hline & Y SCHOOL & & & & \\
\hline & COLLEGE & $6(31.6 \%)$ & $4(21.1 \%)$ & $9(47.4 \%)$ & \\
\hline TYPE OF & NUCLEAR & $13(34.2 \%)$ & $10(26.3 \%)$ & $15(39.5 \%)$ & 0.856 \\
\hline FAMILY & JOINT & $5(33.3 \%)$ & $3(20.0 \%)$ & $7(46.7 \%)$ & \\
\hline $\begin{array}{l}\text { FAMILY } \\
\text { HISTORY }\end{array}$ & NO & $15(33.3 \%)$ & $12(26.7 \%)$ & $18(40.0 \%)$ & 0.686 \\
\hline OF SUICIDE & YES & $3(37.5 \%)$ & $1(12.5 \%)$ & $4 \quad(50.0 \%)$ & \\
\hline
\end{tabular}

\section{Correlation of socio-demographic variables with severity of suicidal attempt}

Depressive patients who are in early adulthood age group (18-40 years) had a more severe suicide attempt than other age group which was statistically significant (p-value-0.026). None of the other variables were statistically significant in their correlation with severity of suicidal attempt (Table 2).

Poisoning was the commonest method of suicide in both men and women. Men had attempted more lethal methods (hanging/electric shock). Almost all women show poisoning as method who attempted suicide. Benzodiazepine poisoning was the most common method used for poisoning by women (Table 2a).

TABLE 2: Correlation of socio-demographic variables with severity of suicidal attempt

\begin{tabular}{|c|c|c|c|c|c|c|}
\hline & & \multicolumn{4}{|c|}{ Suicidal Attempts } & \multirow[t]{2}{*}{ P-Value } \\
\hline & & Absent & Low & Medium & High & \\
\hline \multirow[t]{3}{*}{ Age } & $18-40$ & $17(68.0 \%)$ & $0(0.0 \%)$ & $4(16.0 \%)$ & $4(16.0 \%)$ & 0.026* \\
\hline & $41-60$ & $14(82.4 \%)$ & $0(0.0 \%)$ & $0(0.0 \%)$ & $3(17.6 \%)$ & \\
\hline & $61-65$ & $9(81.8 \%)$ & $2(18.2 \%)$ & $0(0.0 \%)$ & $0(0.0 \%)$ & \\
\hline \multirow[t]{2}{*}{ Gender } & Male & $16(66.7 \%)$ & $2(8.3 \%)$ & $2(8.3 \%)$ & $4(16.7 \%)$ & 0.348 \\
\hline & Female & $24(82.8 \%)$ & $0(0.0 \%)$ & $2(6.9 \%)$ & $3(10.3 \%)$ & \\
\hline \multirow{3}{*}{$\begin{array}{l}\text { Socio } \\
\text { Economic } \\
\text { Status }\end{array}$} & High & $4(80.0 \%)$ & $0(0.0 \%)$ & $1(20.0 \%)$ & $0(0.0 \%)$ & 0.797 \\
\hline & Middle & $14(70.0 \%)$ & $1(5.0 \%)$ & $1(5.0 \%)$ & $4(20.0 \%)$ & \\
\hline & Low & $22(78.6 \%)$ & $1(3.6 \%)$ & $2(7.1 \%)$ & $3(10.7 \%)$ & \\
\hline \multirow{3}{*}{$\begin{array}{l}\text { Marital } \\
\text { Status }\end{array}$} & Married & $30(75.0 \%)$ & $2(5.0 \%)$ & $2(5.0 \%)$ & $6(15.0 \%)$ & 0.660 \\
\hline & Unmarried & $7(70.0 \%)$ & $0(0.0 \%)$ & $2(20.0 \%)$ & $1(10.0 \%)$ & \\
\hline & Widow & $3(100.0 \%)$ & $0(0.0 \%)$ & $0(0.0 \%)$ & $0(0.0 \%)$ & \\
\hline \multirow[t]{3}{*}{ Occupation } & Employed & $17(77.3 \%)$ & $0(0.0 \%)$ & $1(4.5 \%)$ & $4(18.2 \%)$ & 0.579 \\
\hline & Unemployed & $20(76.9 \%)$ & $2(7.7 \%)$ & $2(7.7 \%)$ & $2(7.7 \%)$ & \\
\hline & Student & $3(60.0 \%)$ & $0(0.0 \%)$ & $1(20.0 \%)$ & $1(20.0 \%)$ & \\
\hline \multirow{2}{*}{$\begin{array}{l}\text { Place Of } \\
\text { Living }\end{array}$} & Urban & $11(68.8 \%)$ & $0(0.0 \%)$ & $2(12.5 \%)$ & $3(18.8 \%)$ & 0.516 \\
\hline & Rural & $29(78.4 \%)$ & $2(5.4 \%)$ & $2(5.4 \%)$ & $4(10.8 \%)$ & \\
\hline Education & Illiterate & $9(90.0 \%)$ & $1(10.0 \%)$ & $0(0.0 \%)$ & $0(0.0 \%)$ & 0.611 \\
\hline
\end{tabular}


Clinical and Socio-Demographic Correlates in Depressive Patients with Suicidal Ideas \& Suicidal Attempts

\begin{tabular}{|c|c|c|c|c|c|c|}
\hline & & \multicolumn{4}{|c|}{ Suicidal Attempts } & \multirow[t]{2}{*}{ P-Value } \\
\hline & & Absent & Low & Medium & High & \\
\hline & $\begin{array}{l}\text { Middle } \\
\text { School }\end{array}$ & $6(85.7 \%)$ & $0(0.0 \%)$ & $0(0.0 \%)$ & $1(14.3 \%)$ & \\
\hline & High School & $8(66.7 \%)$ & $0(0.0 \%)$ & $1(8.3 \%)$ & $3(25.0 \%)$ & \\
\hline & $\begin{array}{l}\text { Higher } \\
\text { Secondary } \\
\text { School }\end{array}$ & $5(100.0 \%)$ & $0(0.0 \%)$ & $0(0.0 \%)$ & $0(0.0 \%)$ & \\
\hline & College & $12(63.2 \%)$ & $1(5.3 \%)$ & $3(15.8 \%)$ & $3(15.8 \%)$ & \\
\hline \multirow{2}{*}{$\begin{array}{l}\text { Type Of } \\
\text { Family }\end{array}$} & Nuclear & 30 (78.9\%) & $2(5.3 \%)$ & $1(2.6 \%)$ & $5(13.2 \%)$ & 0.151 \\
\hline & Joint & $10(66.7 \%)$ & $0(0.0 \%)$ & $3(20.2 \%)$ & $2(13.3 \%)$ & \\
\hline \multirow{2}{*}{$\begin{array}{l}\text { Family } \\
\text { History } \\
\text { Of Suicide }\end{array}$} & No & $34(75.6 \%)$ & $2(4.4 \%)$ & $4(8.9 \%)$ & $5(11.1 \%)$ & 0.560 \\
\hline & Yes & $6(75.0 \%)$ & $0(0.0 \%)$ & $0(0.0 \%)$ & $2(25.0 \%)$ & \\
\hline
\end{tabular}

Table 2a: Correlation of gender and method of suicide

\begin{tabular}{|l|l|l|l|l|l|l|l|l|l|}
\hline \multicolumn{2}{|l|}{} & \multicolumn{2}{|l|}{ Method Of Suicide } \\
\cline { 2 - 9 } & $\begin{array}{l}\text { No. Of } \\
\text { Suicide } \\
\text { Attempt } \\
\text { Patients }\end{array}$ & $\begin{array}{l}\text { No } \\
\text { Suicide } \\
\text { Attempt }\end{array}$ & Poison & $\begin{array}{l}\text { Electric } \\
\text { Shock }\end{array}$ & $\begin{array}{l}\text { Hangin } \\
\text { g }\end{array}$ & Burn & $\begin{array}{l}\text { Wris } \\
\text { t Cut }\end{array}$ & Multiple \\
\hline \multirow{2}{*}{ gender } & male & $\begin{array}{l}8 \\
(61.5 \%)\end{array}$ & $\begin{array}{l}16 \\
(66.7 \%)\end{array}$ & $\begin{array}{l}2 \\
(8.3 \%)\end{array}$ & $\begin{array}{l}1 \\
(4.2 \%)\end{array}$ & $\begin{array}{l}3 \\
(12.5 \%)\end{array}$ & $\begin{array}{l}1 \\
(4.2 \%)\end{array}$ & $\begin{array}{l}1 \\
(4.2 \\
\%)\end{array}$ & $0(0.0 \%)$ \\
\cline { 2 - 10 } & female & $\begin{array}{l}5 \\
(38.5 \%)\end{array}$ & $\begin{array}{l}24 \\
(82.8 \%)\end{array}$ & $\begin{array}{l}4(13.8 \%) \\
(0.0 \%)\end{array}$ & $\begin{array}{l}0 \\
(0.0 \%)\end{array}$ & $\begin{array}{l}0 \\
(0.0 \%)\end{array}$ & $\begin{array}{l}0 \\
(0.0 \\
\%)\end{array}$ & $1 .(3.4 \%)$ \\
\hline
\end{tabular}

Correlation of history of previous suicide attempts with severity of suicide ideation \& current severity of suicidal attempt

Depressive patients who had attempted suicide before had more severe suicidal ideas than patients without previous suicidal attempt which was statistically significant p-value- $\mathbf{0 . 0 1}$ ).

Patients who had past history of suicide attempt had higher severity of current suicide attempt which was statistically highly significant (p-value-0.000). (Table 3)

Table 3: Correlation of suicide attempt history with severity of suicide ideation and suicidal attempt

\begin{tabular}{|l|l|l|l|l|l|}
\hline \multirow{2}{*}{} & & \multicolumn{3}{|l|}{ SUICIDAL IDEATION SEVERITY } & \multirow{2}{*}{ P-VALUE } \\
\cline { 3 - 5 } & & ABSENT & MILD & SEVERE & \\
\hline \multirow{2}{*}{$\begin{array}{l}\text { PREVIOUS } \\
\text { SUICIDE }\end{array}$} & NONE & $18(45.0 \%)$ & $12(30.0 \%)$ & $10(25.0 \%)$ & \multirow{2}{*}{ 0.01* } \\
\cline { 2 - 5 } ATTEMPTS & ONCE & $0(0.0 \%)$ & $1(8.3 \%)$ & $11(91.7 \%)$ & \\
\cline { 2 - 5 } & MORE & $0(0.0 \%)$ & $0(0.0 \%)$ & $1(100.0 \%)$ & \\
& THAN ONCE & & & & \\
\hline
\end{tabular}

(C) The International Journal of Indian Psychology, ISSN 2348-5396 (e)| ISSN: 2349-3429 (p) | 93 
Clinical and Socio-Demographic Correlates in Depressive Patients with Suicidal Ideas \& Suicidal Attempts

\begin{tabular}{|l|l|l|l|l|l|l|}
\hline \multicolumn{2}{|c|}{} & \multicolumn{3}{|l|}{ SUICIDAL ATTEMPT SEVERITY } & \multirow{2}{*}{ P-VALUE } \\
\cline { 2 - 7 } \multicolumn{2}{|c|}{} & ABSENT & LOW & MEDIUM & HIGH & \multirow{2}{*}{ 0.000* } \\
\hline \multirow{3}{*}{$\begin{array}{l}\text { PREVIOUS } \\
\text { ATTEMPT }\end{array}$} & NONE & $\begin{array}{l}40 \\
(100.0 \%)\end{array}$ & $0(0.0 \%)$ & $0(0.0 \%)$ & $0(0.0 \%)$ & \\
\cline { 2 - 7 } & ONCE & $0(0.0 \%)$ & $2(16.7 \%)$ & $4(33.3 \%)$ & $6(50.0 \%)$ & \\
\cline { 2 - 6 } & $\begin{array}{l}\text { MORE } \\
\text { THAN } \\
\end{array}$ & $0(0.0 \%)$ & $0(0.0 \%)$ & $0(0.0 \%)$ & $1 \quad(100.0 \%)$ & \\
\hline
\end{tabular}

\section{Correlation of severity of depression with suicidal ideation}

The higher the severity of depression on HAMD rating scale, the more severe was the suicidal ideation and this was statistically significant. $(p=0.020)$ (Table 4)

Table 4: Severity of depression and suicidal ideation

\begin{tabular}{|c|c|c|c|c|c|}
\hline & & SUICIDAL & [DEATION & & P - VALUE \\
\hline \multirow{4}{*}{$\begin{array}{l}\text { SEVERITY } \\
\text { OF } \\
\text { DEPRESSION }\end{array}$} & & ABSENT & MILD & SEVERE & \\
\hline & MODERATE & $4(100.0 \%)$ & $0(0.0 \%)$ & $0(0.0 \%)$ & \multirow[t]{3}{*}{$0.020 *$} \\
\hline & SEVERE & 3 (42.9\%) & $3(42.9 \%)$ & 1 (14.3\%) & \\
\hline & $\begin{array}{l}\text { VERY } \\
\text { SEVERE }\end{array}$ & $11(26.2 \%)$ & $10(23.8 \%)$ & 21 23.8\%) & \\
\hline
\end{tabular}

\section{Correlation of severity of suicide idea and severity of suicide attempt}

Depressive patients who had higher suicidal ideas had attempted suicide with higher intentionality $(\mathrm{R}$ value $=0.364$, $\mathrm{p}$-value-0.007) which was statistically significant.

\section{DISCUSSION}

\section{Incidence of suicidal ideation and attempt in the sample}

The incidence of severe suicidal ideation on the MSSI rating scale in our sample was $41.5 \%$ whereas in the sokero et al study, $58 \%$ of all patients had experienced suicidal ideation. This could be because in the latter study they had also included patients having substance use in their sample. These findings further stresses on the importance of screening for suicidal ideation in patients with major depressive disorder.

The incidence for attempted suicide in the sample was $24.5 \%$ which is much higher than the study by sokero et al which had reported incidence of $15 \%$. It is also higher than incidence reported in Indian study by Srivastava et al (16.6\%). This higher incidence in our sample could be because of fact that majority (92.4\%) of the patients in the sample had severe depression on HAMD rating. 


\section{Clinical and Socio-Demographic Correlates in Depressive Patients with Suicidal Ideas \& Suicidal Attempts}

In the other studies they have looked into only the incidence of suicidal thoughts and attempts, whereas in our study the correlation of socio demographic and clinical variables with the severity of suicidal ideation and attempts were analyzed.

\section{Gender}

Though there were higher no. of males with severe suicidal ideation (50\%) compared to females (34.5\%), but this difference was not statistically significant. In the cross-national study by Weissman et al, also there was no statistical significant difference in the suicidal ideation between the genders. In other Indian studies also no significant difference has been identified.

When taking the suicidal attempters group, more number of males (33.3\%) had attempted suicide than females (17.2\%). Even when considering severity of suicidal attempt across from low to high it were the males who were consistently higher represented across the severity of the attempt but not statistically significant. This is comparable to findings by Narang et al (sample size 208), that found that the prevalence of suicidal attempt was high among males with psychiatric illness (ICD 10 criteria), when compared to females. In contrast the western studies have shown that there is significantly higher risk in women than in men for suicidal attempt. Our study is unique in that other Indian or western studies have not compared the correlations with the severity of suicidal attempts and ideation in patients with major depressive disorder without co morbidities.

\section{Socio economic status and place of living}

In our sample the lower socioeconomic status patients were represented the most (52.8\%), followed by the middle class (37.7\%) and higher socioeconomic status were only $9.4 \%$. When considering severe suicidal ideation, all the 3 groups were comparable. Whereas compared to the high and middle income groups, the low income group had most persons with suicidal ideation but these differences were not statistically significant. But when considering rural versus urban, though rural population had the most representation (69.8\%), the no. of patients who had the most severe suicidal ideation were seen in the urban group (56.3\%) versus the rural group (35.1\%), but again this was not statistically significant. But when looking at patients with suicidal attempts the proportion were almost equal in the rural versus urban and in the various socioeconomic strata. This finding is comparable to findings from Narang et al

\section{Marital, Literacy and employment}

In our sample the proportion of patients representing unmarried population was very less (24.5\%) compared to married (75.5\%), thus not allowing for meaningful comparison. When considering the employment status, there was no statistically significant difference in the severity of suicidal ideation with both employed and unemployed having proportions of $36.4 \%$ and $46.2 \%$ respectively showing severe levels of suicidal ideation. But when we compared the 
severity of suicidal attempt, the proportion of employed patients (18.2\%) had more severe suicidal attempt than unemployed (7.7\%), but the difference did not reach statistical significance. Also when comparing illiterate versus literate patients, the severity of suicidal ideation was comparable across the levels of literacy studied. But in severity of suicidal attempt, the proportion of literate patients had more severe attempt than the illiterate group (in which only 1 patient had a low severe suicidal attempt). These findings are comparable to the Srivastava et al study $^{7}$ that found that more educated patients in the group were more likely to attempt suicide. However, Kessler et al had found that poorly educated subjects were more vulnerable to suicidal attempts, but here we also have to note that this study included various psychiatry diagnoses whereas the srivastava et al study included only major depressive disorder patients similar to our current study.

\section{Correlation of Type of family and family h/o suicide with severity of suicidal idea and attempt}

Though the proportion of patients who had severe suicidal ideation was slightly higher in joint family (46.7\%) group compared with nuclear family (39.5\%), it was not statistically significant. Concordantly the proportion of patients with suicidal attempt were also higher in joint family group (33.3\%), compared to the nuclear family group. But these differences were not statistically significant same as even reported in study by Narang et al, in which they had a larger sample size (100).

Only 8 patients out of the whole sample had reported family history of suicide. But the proportion of these patients with severe suicidal ideation was 50\%, which was slightly higher than the patients without family history of suicide who had severe suicidal ideation (40\%). Similarly even though the sample size was very less in the patients with family history of suicide, $25 \%$ of them had severe suicidal attempt which was greater than when compared with the patients without family history in whom the proportion of patients with severe suicidal attempt was only $11 \%$. But again these differences were not statistically significant. Indian studies have shown that family history of attempted suicide or completed suicide was common among patients suffering from depression

\section{Correlation of history of previous suicide attempts with severity of suicide ideation \& attempt}

12 patients (22.6\%) in the sample had at least one history of previous suicidal attempt and one patient had previous history of more than once. The proportion of patients who had severe suicidal ideation was higher in the group of patients with previous suicidal attempt (92.3\%) than those without (25\%). This difference was statistically significant ( $\mathrm{p}=0.01)$

All the patients who attempted suicide during the current episode (13) had a previous history of suicide attempt, compared to those without suicide attempt during current episode that had none. This difference was also statistically significant $(p=0.000)$. Thus from this study we further confirm to the understanding that the history of previous suicide attempt in major depressive 
disorder appears to be a major risk factor for suicidal attempt or severe suicidal ideation during the subsequent episodes also. This finding has been replicated in previous studies that have found attempted suicide to be one of the predictors of future suicide. Studies in India shows that about $18 \%$ of suicide victims had a previous suicide attempt.

\section{Correlation between Severity of depression and suicide ideation and attempt in the sample}

All the patients with suicidal attempt in the sample were patients who had very severe depression on HAMD rating scale. Suicidal attempt has been found to be positively correlated with severity of depression in other Indian studies also ${ }^{15}$. Also the severity of suicidal ideation was positively correlated with the severity of depression on HAMD rating scale. $(p=0.020)$

\section{Correlation of severity of suicide idea and severity of suicide attempt}

Depressive patients who had higher suicidal ideas in our sample had attempted suicide with higher intentionality which was statistically significant $(R$ value $=0.364, p$-value-0.007). Study by Kessler et al had shown that cumulative probabilities for transition from suicidal idea to plan were $34 \%$ and for transition from plan to attempt was $72 \%{ }^{21}$. But they had included various co morbidities and whereas our study is specifically on major depressive disorder and also we have not differentiated between suicidal ideators and suicidal plan in our study. We have not analyzed the individual factors in the rating scales for suicidal ideation and attempts across the various variables studied.

\section{Strengths and limitations}

This was a cross sectional study in the inpatient ward during the patient's current depressive episode thus we were able to assess in cross sectionally, the suicidal behavior without any recall bias. In our sample the patients with severe depression was represented adequately and there were no dropouts, thus were able to assess suicidal behavior true to our study objective. We used validated scales and we stuck to a homogenous sample analyzing subjects with diagnosis of major depressive disorder alone and excluded subjects with substance abuse and other psychiatric co morbidities that could influence suicidal behavior such as personality disorders or adjustment disorder etc.

Some of the limitations of our study are that the researcher may have been biased when assessing the suicidal behavior as the severity rating was also done by the same interviewer. Since it was a cross sectional study we have not analyzed the course of the suicidal behavior in these group of patients and their response to treatment. We have not studied the correlation of the presence of any recent life events with severity of suicidal behavior. Further studies are needed to compare whether the correlations with severity of suicidal behavior differ in other psychiatric illnesses like schizophrenia, bipolar disorder, personality disorder etc. 


\section{CONCLUSION}

Severity of depression was correlated with severity of suicidal ideations as well as severity of suicidal attempts. In addition to identifying suicidal behavior, the assessment of severity of suicidal ideations and past suicidal attempts is of great importance in identifying high risk patients presenting with major depressive disorder.

\section{Acknowledgments}

We thank our institution, PSG Institute of Medical Science \& Research, Coimbatore and our department of Psychiatry, for allowing us to conduct this research and our colleagues for the great insights that helped in improving the manuscript.

Conflict of Interests: The author declared no conflict of interests.

\section{REFERENCES}

A.S. Srivastava, Rakesh Kumar; Suicidal ideation and attempts in patients with major depression: Sociodemographic and clinical variables; Indian Journal of Psychiatry 2005;47:225-228

Aponte (1969) Epidemiological aspects of suicide and attempted suicide. Proceeding of the 5th International Conference for Suicide Prevention (Ed.) Fox, R., Vienna.

Beck, R.W Morris, J.B, and Beck, A.T (1974) cross- validation of the suicidal intent scale. Psychological reports, 34, 445-446

Bhatia MS, Aggarwal NK, Aggarwal BB. Psychosocial profile of suicide ideators, attempters and completers in India. Int J Soc Psychiatry 2000;46:155-63.

David, L. (1970) Relationship between attempted suicides and completed suicides. Psychological Reports, 27(3), 719-722.

E C Harris and B Barraclough; Suicide as an outcome for mental disorders. A meta-analysis; BJP 1997, 170:205-228

Gelder, M., Gath, D., Mayou, R. \& Cowen, P. (1996). Oxford Textbook of Psychiatry, 3rd edition: Oxford University Press, 414-455.

Harris EC, Barraclough B: Excess mortality of mental disorder. Br J Psychiatry. 1998, 173: 1153. 10.1192/bjp.173.1.11

Hawton K, Fagg J. Suicide, and other causes of death, following attempted suicide. $\mathrm{Br} J$ Psychiatry 1988;152:359-66.

Kessler RC, Borges G, Walters EE. Prevalence of and risk factors for lifetime suicide attempts in the National Comorbidity Survey. Arch Gen Psychiatry 1999;56:617-26.

M.M. Weissman et al; Prevalence of suicide ideation and suicide attempts in nine countries; Psychological Medicine, 1999, 29, 9-17

Malone KM, Oquendo MA, Haas GL, et al. Protective factors against suicidal acts in major depression: Reasons for living. Am J Psychiatry 2000;157:1084-8. 


\section{Clinical and Socio-Demographic Correlates in Depressive Patients with Suicidal Ideas \& Suicidal Attempts}

Mathers C, Fat DM, Boerma JT, et al. The global burden of disease: 2004 update; Geneva, Switzerland: World Health Organization; 2008.

Nandi, D.N., Banerjee, G \& Boral, G.C. (1978). Suicide in West Bengal; Indian Journal of Psychiatry, 20, 155-60.

Narang RL, Mishra BP, Mohan N. Attempted suicide in Ludhiana. Indian J Psychiatry 2000;42:83-7.

National Crime Records Bureau. Accidental Deaths and Suicides in India 2008. Ministry of Home Affairs, Government of India New Delhi; 2010.

P.N Suresh Kumar; An Analysis of Suicide Attempters Versus Completers in Kerala; Indian Journal of Psychiatry, 2004, 46(2)144-149.

Pettit JW et al; Factor structure and psychometric properties of the Modified Scale for Suicidal Ideation among suicidal youth; Depress Anxiety. 2009;26(8): 769-74.

Phillips, M.R., Xianylun, Li \& Zhang, Y (2003) Suicide rate in China, 1995- 1999. In: Vijayakumar, L. Suicide Prevention - Meeting the challenge together, Orient Longman, 214-231.

Rao VA; Attempted suicide; Indian J Psychiatry; 1965;7: 253-64

Singh, A.N. (1972). The study of 38 cases of suicides and attempted suicides and their causation in teenagers. Proceedings of the 6th International Conference for Suicide prevention (Ed.). Litman, Mexico.

Sokero TP, Melartin TK, Rytsala HJ, et al. Suicidal ideation and attempts among psychiatric patients with major depressive disorder. J Clin Psychiatry 2003;64:1094-100.

Srivastava S, Kulshreshtha N. Expression of suicidal intent in depressives. Indian J Psychiatry 2000;42:184

Thomas E. Joiner Jr., M. David Rudd et al The Modified Scale for Suicidal Ideation: Factors of Suicidality and Their Relation to Clinical and Diagnostic Variables; Journal of Abnormal Psychology 1997. Vol. 106, No. 2, 260-265

Vijayakumar L; Indian research on suicide; Indian J Psychiatry; 2010;52:S291-6.

World Health Organisation; WHO Fact sheet; reviewed September 2016; http://www.who.int/mediacentre/factsheets/fs398/en/

How to cite this article: Ummer S, Sugathan S (2017), Clinical and Socio-Demographic Correlates in Depressive Patients with Suicidal Ideas \& Suicidal Attempts, International Journal of Indian Psychology, Volume 4, Issue 2, No. 89, ISSN:2348-5396 (e), ISSN:2349-3429 (p), DIP:18.01.089/20170402, ISBN:978-1-365-74162-3 